\title{
Neurodegenerative disorders, bullous pemphigoid and psoriasis: a comparative study in ethnic Poles indicates that Parkinson's disease is more relevant to bullous pemphigoid
}

\author{
Paweł Bartkiewicz' ${ }^{1}$ Justyna Gornowicz-Porowska ${ }^{1}$, Paweł P. Pietkiewicz'1, Anna Świrkowicz', \\ Monika Bowszyc-Dmochowska², Marian Dmochowski ${ }^{1}$ \\ ${ }^{1}$ Autoimmune Blistering Dermatoses Section, Department of Dermatology, Poznan University of Medical Sciences, Poznan, Poland \\ ${ }^{2}$ Cutaneous Histopathology and Immunopathology Section, Department of Dermatology, Poznan University of Medical Sciences, \\ Poznan, Poland
}

Adv Dermatol Allergol 2017; XXIV (1): 42-46

DOI: https://doi.org/10.5114/ada.2017.65619

\begin{abstract}
Introduction: Bullous pemphigoid (BP) is an autoimmune blistering dermatosis of the elderly with autoimmunity to hemidesmosomal proteins, BP180 and BP230, which are expressed also in neuronal tissue.

Aim: The aim here was to retrospectively compare the prevalence of neurodegenerative disorders (ND), particularly Parkinson's disease (PD), unspecified conditions manifesting as dementia and stroke, in two groups of ethnic Poles, with BP and with psoriasis (Ps), in order to obtain data whether BP is more prone to coexist with ND than Ps in the elderly. Psoriasis was chosen in this comparative study as it was considered to be a paradigm of cutaneous disease with systemic manifestations.

Material and methods: The available medical records of 96 BP patients and 149 Ps patients over 70 years of age were analyzed for the presence of ND.

Results: There were no statistically significant differences in prevalence of ND without specifying the type and ND types analyzed between BP and Ps groups, except for a higher prevalence of PD in the BP group.

Conclusions: Thus, regarding population aging and increasing incidence and prevalence of BP corresponding with that phenomenon in various ethnicities, it appears justified to expand studies of a possible immunopathogenic relationship, appearing to be PD-related, between BP and ND.
\end{abstract}

Key words: pemphigoid, bullous, psoriasis, neuropathology, autoantigens.

\section{Introduction}

Neurodegenerative disorders (ND) are among the most serious health problems of the current population. Most of these disorders become more common with advancing age, including Parkinson's disease (PD), unspecified conditions manifesting as dementia (UnsD), stroke, and others. The prevalence of ND is growing as the population ages, with simultaneously growing economic and social costs. Some ND have clear genetic causes, whereas others involve complex interactions of genetic and environmental factors.

Bullous pemphigoid (BP) is the immunobullous disease due to circulating antibodies that bind two components of keratinocyte hemidesmosomal proteins; the two antigens are type XVII collagen/BPAG2 (BP180) and
BPAG1 (BP230). A stereotypical clinical manifestation of this clinically vastly heterogeneous dermatosis comprises pruritic papular and/or urticarial lesions with large tense bullae (subepidermal blisters with abundance of eosinophils histologically) [1, 2]. BP is the most common bullous autoimmune disease occurring more commonly in the elderly-octogenarians and older. A French study revealed a 3-fold increase in the annual prevalence of BP over the last 15 years, with 21.7 new cases per million inhabitants [3]. BPAG1, i.e. bullous pemphigoid antigen 1, encoded by the dystonin gene (Dst) [4], is a member of the plakin family. This protein family provides the structure of cytoskeletal networks. Multiple transcription paths and alternative splicing of Dst results in three major tissue-specific BPAG1 isoforms, BPAGle ( 300 kDa) found in stratified

Address for correspondence: Paweł Bartkiewicz, Autoimmune Blistering Dermatoses Section, Department of Dermatology, Poznan University of Medical Sciences, 49 Przybyszewski St, 60-355 Poznan, Poland, phone: +48 61869 13 67, e-mail: lu.bartkiewicz@gmail.com Received: 5.01.2016, accepted: 7.02.2016. 
epithelia, BPAG1a ( 600 kDa) expressed in neurons and BPAG1b ( 800 kDa) expressed in both skeletal and cardiac muscles [5]. The role of various BPAG1 isoforms is depicted by cases of genetic defects of BPAG1 (natural mutations or engineered inactivation of Dst) in mice resulting in dystonia musculorum. In humans, a pathogenic mutation affecting the BPAG1a/b results in sensory autonomic neuropathy. Dst-null mice showed also discrete signs of skin blistering due to the absence of BPAG1e. In humans, by analogy, homozygous nonsense DST mutations affecting BPAG1e result in epidermolysis bullosa variant [6]. The common ectodermal origin of the skin and entire central nervous system results in analogous molecular isoforms of structural proteins and therefore may implicate similar pathogenetic background for $\mathrm{cu}-$ taneous and neurological disorders. Claudepierre et al. have shown the expression of BPAG1 and BPAG 2 in the adult and developing retina. Interestingly, both molecules are expressed in rods, whereas only BPAG2 is expressed in cones [7].

Recent studies have shown that patients with BP are more likely to suffer from neurological and psychiatric diseases (unipolar and bipolar disorders), particularly prior to the diagnosis of BP. It was reported that BP is associated with neurodegenerative diseases, and in such cases usually a neurological manifestation can precede cutaneous involvement. The burden of these neurodegenerative diseases includes PD, UnsD, stroke epilepsy, multiple sclerosis or neurosyphilis [8-10]. According to a British study, BP followed neurologic diseases in most patients ( 26 of 36 patients $-72 \%$ of them), with a median interval of 5.5 years [11].
Psoriasis (Ps) affects up to $2 \%$ of the Western population, is considered as a chronic disorder with a polygenic predisposition combined with triggering factors such as infections, medication, or trauma. Maintenance of psoriatic lesions is considered an ongoing autoreactive immune response $[1,2]$. Being widely recognized as a systemic inflammatory condition, psoriasis is an immune-mediated T-cell-driven (including TNF- $\alpha$ and IL-23) inflammatory disease of the skin, joints and involving the cardiovascular system $[1,12]$. The increased cardiovascular risk in Ps patients includes stroke [13-15]. However, there are reports suggesting that a high prevalence of psychiatric disorders like depression in Ps could be a component of systemic inflammation and not only the psychosomatic consequence [12]. Some reports show that patients with mild to severe plaque Ps develop concomitant features of mild cognitive impairment [16]. Numerous cases of Ps preceding autoimmune blistering dermatoses, particularly BP, were reported in the literature, including a report from our university department [17]. The term "psoriasis bullosa acquisita" [18] suggested for Ps coincident with the subepidermal autoimmune blistering dermatosis with IgG antibodies to antigen p200, i.e. laminin $\gamma 1$, nature of which was at that time unknown, being wrongly speculated that it might be related to type VII collagen, an autoantigen in epidermolysis bullosa acquisita, is misleading and as such is not in use now as Ps can precede any subepidermal and, in fact, any intraepidermal autoimmune blistering dermatosis.

Recently, we have seen in our department two elderly males with Ps lasting many decades in whom coexisting BP was diagnosed after considering the clinical picture and results of molecular diagnostic tests (Figure 1). On
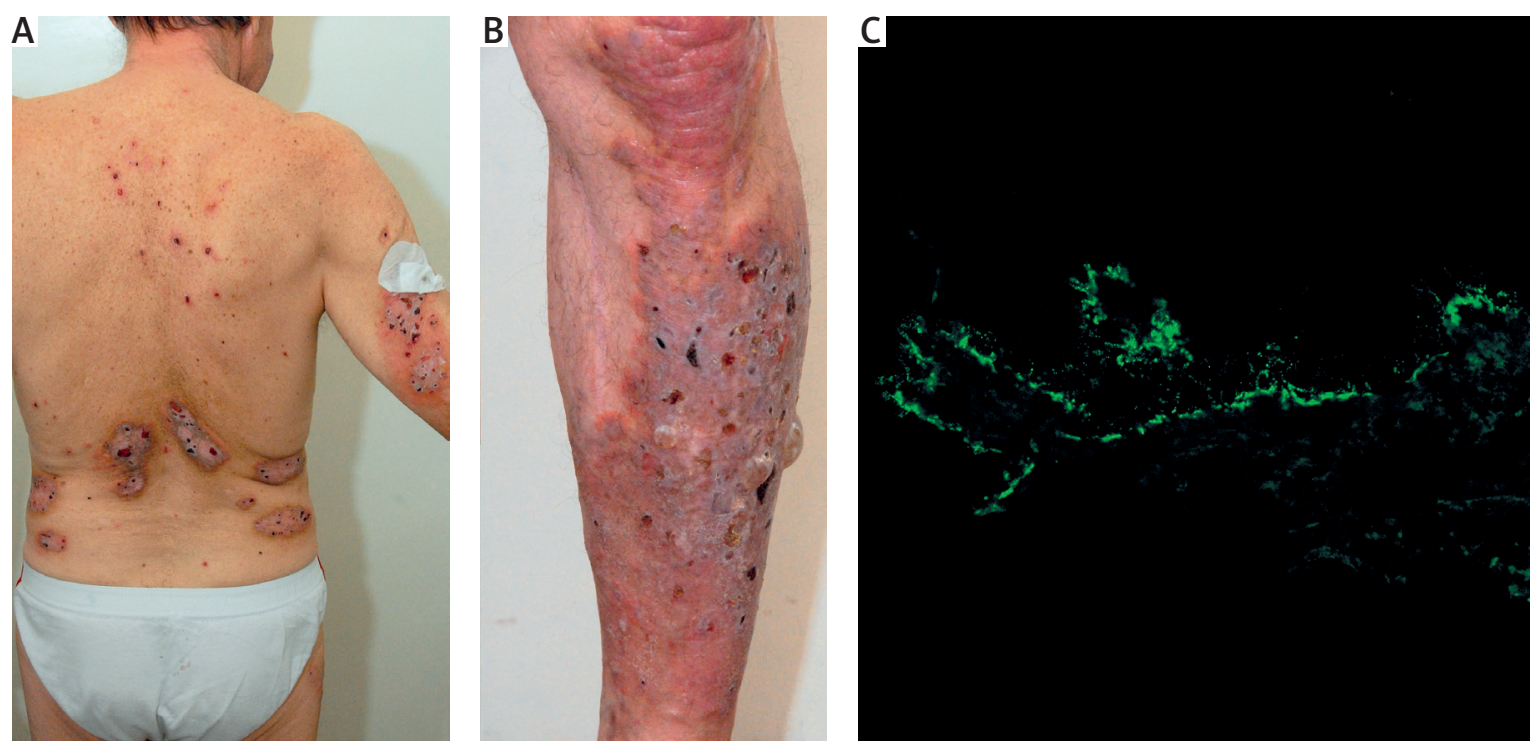

Figure 1. An elderly male with long-lasting Ps coexistent with newly developed BP showing psoriatic partially eroded plaques, particularly severe on the lumbar skin (A), and tense blisters superimposed on a psoriatic plaque on the shin (B) in whom direct immunofluorescence on nonlesional peribullous skin using laser scanning confocal microscopy for visualization demonstrated IgG4 deposits along the dermal-epidermal junction (C) and ELISA revealed elevated levels of serum IgG antibodies to both BPAG1 and BPAG2 (both levels above $200 \mathrm{RU} / \mathrm{ml}$, both cut-off levels $20 \mathrm{RU} / \mathrm{ml}$ ) 
Table 1. ND in BP and Ps patients studied

\begin{tabular}{lcc}
\hline Parameter & BP group & Ps group \\
\hline Patients & 96 & 149 \\
\hline Gender & 59 female, 37 male & 76 female, 73 male \\
\hline Non-ND & 69 & 107 \\
\hline ND & 27 & 42 \\
\hline UnsD & 5 & 14 \\
\hline Stroke & 12 & 15 \\
\hline PD & 5 & 1 \\
\hline Other ND & 5 & 12 \\
\hline $\begin{array}{l}\text { More than } \\
\text { one ND }\end{array}$ & 3 & 1 \\
\hline
\end{tabular}

$B P$ - bullous pemphigoid, PS - psoriasis, ND - neurodegenerative disease, UnsD - unspecified conditions manifesting as dementia, PD - Parkinson's disease.

the ground of such cases it can be speculated that inflammation observed in Ps patients can trigger autoimmunity leading to clinically overt bullous dermatosis by exposing hidden epitopes, particularly of structural proteins building the dermal-epidermal junction.

\section{Aim}

The aim of our study was to retrospectively compare, analyzing medical records available, the prevalence of ND in two groups of patients, with BP and with Ps, in order to obtain data whether BP is more prone to coexist with ND than Ps in the elderly. Psoriasis was chosen in this comparative study as it was considered to be a paradigm of cutaneous disease with systemic manifestations and the coexistence of Ps with BP, a disease frequently coexistent with systemic illnesses, indicates that they might share pathogenic pathways, both cutaneous and extracutaneous. This study design comparing two dermatoses that share inflammation and autoimmunity phenomena was hoped to provide data more relevant to BP than studies merely comparing BP and healthy population.

\section{Material and methods}

Medical histories of 96 BP patients diagnosed at a molecular level with direct immunofluorescence, indirect immunofluorescence on monkey esophagus and/ or multiparametric mosaic (Euroimmun, Germany) and ELISA for IgG antibodies to BPAG1 and BPAG2 (Euroimmun, Germany) (27 BP patients with ND (BP + ND) and 69 BP patients without ND (BP-ND)), and 149 Ps patients over 70 years of age (42 Ps patients with ND (Ps + ND) and 107 Ps patients without ND (Ps-ND)) hospitalized in the university dermatology department between December 2006 and July 2015 (BP) and between January 2002 and May 2015 (Ps) were reviewed for the presence of ND records. Neurodegenerative disorders included PD, UnsD, stroke, hear loss, tinnitus, blindness, vertigo, epilepsy, neurosyphilis, and also systemic sclerosis.

\section{Statistical analysis}

Sex differences, differences in prevalence of ND and stroke between groups were examined with Pearson's $\chi^{2}$ test, whereas differences in prevalence of PD, UnsD and number of patients suffering from more than one ND were assessed with Fisher's exact test (2-sided). Statistical analysis was performed with "Statistica PL 10.0" (StatSoft Inc., Tulsa, Oklahoma, USA). A $p<0.05$ was arbitrarily considered statistically significant.

\section{Results}

The detailed results are shown in Table 1. There was a significant difference in prevalence of PD (BP vs. Ps: $5.21 \%$ vs. $0.67 \% ; p=0.031$.

Statistical analysis revealed insignificant differences in prevalence of ND (without specifying the type) (BP vs. Ps: $28.13 \%$ vs. $28.19 ; p=0.992$ ), stroke (BP vs. Ps: $12.5 \%$ vs. $10.07 \%$; $p=0.637$ ), UnsD (BP vs. Ps: $5.21 \%$ vs. $9.40 \%$; $p=0.181)$ and number of patients with more than one ND (BP vs. Ps: $3.13 \%$ vs. $0.67 \%$; $p=0.292$ ) between groups. There were no statistically significant sex differences between groups ( $p=0.108)$.

\section{Discussion}

The advancing age of our population and health disorders related to ageing become a socioeconomic problem. Thus, studies concerning a disease mechanism of BP and ND should be considered as an urgent need.

A study suggested that the clinical picture, conceivably including ND, of solely anti-BPAG2 IgG positive BP patients may differ from that seen in solely anti-BPAG1 IgG positive patients (dubbed anti-BP230 BP) or in patients with coexisting IgG antibodies to both BPAG1 and BPAG2 [19]. Nevertheless, in our issue-probing retrospective immunopathologic study, the levels of serum IgG antibodies to either BPAG1 or BPAG2 have not influenced the occurrence of ND in BP [20]. Still, ND can be more prevalent in BP patients having autoimmunity to both BPAG1 and BPAG2 particularly in the cerebrospinal fluid and this possibility remains to be verified. Data from studies on sera of PD patients and non-parkinsonian dementia patients provided a novel insight into the association between BP and ND. Patients with PD, or dementia but without BP, had increased levels of autoantibodies against the BPAG2. Almost a third of patients (14 of 50 patients $-28 \%$ ) with either PD (25 patients) or nonparkinsonian dementia (25 patients) had autoantibodies to BPAG2 in their sera. Of these, only 1 of 25 dementia sera and none of the PD sera had autoantibodies against pathogenic NC16A epitope of BPAG2 [21].

The process of immunosenescence might be considered as a link between BP and ND. Immunosenescence is 
the age-associated dysregulation of immunity, characterized by domination of proinflammatory mediators and a switch to Th2-type responses, with a parallel decrease in adaptive immunity. Flow cytometric evaluation of peripheral blood of untreated BP patients revealed that BP patients had an increase in biomarker of "immune cell exhaustion" (CD8 ${ }^{+} /$CD28- lymphocytes) compared to age-matched controls or young controls. This condition significantly increases the risk of tissue degeneration in BP, Ps as well as ND [22]. On the mouse model, neurological degeneration and dystonia musculorum were preceded by genetic alteration of BPAGla which resulted in accumulation of intermediate filaments in motor neurons. The intermediate filaments' accumulation might be an initiating factor for loss of the tolerance process to BPAG1a and leading to cross-intolerance of epidermal BPAG1e [23].

Coincidentally, autoreactivity to the substantia nigra, a midbrain region of critical importance in pathogenesis of PD, a disease implicated here to be BP-related ND, of the rat and human brain was tested by indirect immunofluorescence performed with antibodies to tyrosine hydroxylase $(\mathrm{TH})$ and patient sera. Autoantibodies in the sera of PD patients co-localized to the TH positive neurons in rat brain, as did from patients with both BP and PD. Antibodies from controls were unable to bind to substantia nigra. Staining of substantia nigra in human brain confirmed these findings [21].

In our study, the prevalence of ND in a group of BP patients $(28.13 \%)$ seems to be within the range of results presented in recent studies from Europe and Brazil: 22-46\% [11, 23-26]. The genetic and environmental factors may strongly influence differences between studies. On the other hand, some BP-ND patients remain underdiagnosed, as the dermatologist may marginalize non-dermatological conditions or simply neglect them in medical records. This situation may lead to a decrease in the number of patients which underwent the evaluation. Also the awareness about the linkage might be still low among dermatologists omitting the neurological conditions and leading to unintentional misinformation.

The prevalence of a cerebrovascular incident in BP patients (12.5\%) from our department was similar to the one reported in the French group study (15\%), but lower than in the British study $(44.4 \%)[11,23,25]$. It is possible that the above factors and high death rate observed in stroke patients in Poland might play a role in the number of reported cerebrovascular incidents.

Parkinson's disease was present in $5.21 \%$ of our BP patients in contrast with $9 \%$ in the French study, and $30 \%$ in the British study $[11,23,25]$. Parkinson's disease rate in Ps patients was low $(0.67 \%)$, interestingly compared with an increased risk of the disease in Ps patients reported in a Taiwanese 5 years' follow-up study [27].

The UnsD rate in the studied BP group reached 5.21\%. A report (population-based study) for Midwestern Poland estimated that dementia was observed in $5.7 \%$ of people above 65 years [28] in contrast to 13\% in the United Kingdom [10]. This result has fitted within the range of the epidemiologic data in that geographic region and corresponds with our findings.

Both BP and neurological diseases are more common with the increasing age. In order to explore a potential causative relationship between neuronal damage and onset of BP, Langan et al. repeated analysis looking for onset of the neurological disease after the diagnosis of BP. In this situation no association was seen, supporting the contention that neuronal antigen exposure may be causatively involved in the subsequent development of BP in patients with the neurological disease [24]. According to that finding, it is also not expected that the dementia rate in the BP group should be higher than in general population (adjusted for age). Interestingly, the rate of dementia in our Ps patients group was higher, albeit insignificantly, (9.4\%) than in the BP group (5.21\%), however Ps patients were above 70 years of age. The study by Langan et al. revealed a highly increased risk of developing BP - threefold in patients with PD and dementia, and twofold in patients with stroke and epilepsy, comparing to healthy controls [24].

Understanding the basic pathophysiology of BP is important because of potentially life-threatening course of this dermatosis, particularly in overtreated debilitated elderly persons. Excitingly, autoimmune encephalitides [29], such as anti-N-methyl-D-aspartate receptor encephalitis which even was graphically depicted in a bestselling nonfiction book available in translations as well [30], can conceivably trigger smoldering intermolecular epitope spreading phenomena leading to the development of BP type autoimmunity, first in the brain and then in the skin in individuals undergoing immunosenescence and bearing underlying putative BP-prone mutations in Fc receptors on neutrophils and eosinophils migrating to the skin. Expanding knowledge of the immunology of ND, by combining experimental and laboratory approaches benefitting from basic facts-establishing epidemiological studies [31], might lead to developing of new treatments for both conditions neurological and cutaneous.

\section{Conclusions}

As there were no statistically significant differences in the prevalence of ND without specifying the type and ND types analyzed between groups, except for a higher prevalence of PD in the BP group, it seems that the majority of pathogenic pathways of ND is common for both BP and Ps. Regarding population aging and an increasing incidence and prevalence of BP corresponding with that phenomenon in various ethnicities, it appears justified to expand studies of a possible immunopathogenic connection, appearing to be PD-related, between BP and ND. 


\section{Acknowledgments}

This study was partly funded from the grant of the Polish Ministry of Science and Higher Education 0127/ IPI/2015/73. Biochip indirect immunofluorescence mosaic was obtained from Euroimmun (Germany) within a framework of a multicenter project: "A European prospective study on serum antibodies against target antigens of bullous autoimmune diseases and genetic susceptibility".

The laser scanning confocal fluorescence microscopy image (Figure $1 \mathrm{C}$ ) was taken at the Laboratory of Electron and Confocal Microscopy, Adam Mickiewicz University in Poznan.

\section{Conflict of interest}

The authors declare no conflict of interest.

\section{References}

1. Bolognia JL, Schaffer JV, Duncan KO, Ko CJ. Vesiculobullous disease. In: Dermatology Essentials. Bolognia JL, Schaffer JV, Duncan KO, Ko CJ (eds.). Elsevier-Saunders 2014; 223-8.

2. Wolff K, Johnson RA, Saavedra AP. Genetic and acquired bullous diseases. In: Fitzpatrick's Color Atlas and Synopsis of Clinical Dermatology. $7^{\text {th }}$ ed. Sydor AM, Davis KJ (eds.). Mc Graw Hill Education, USA 2013; 107-9.

3. Joly P, Baricault S, Sparsa A, et al. Incidence and mortality of bullous pemphigoid in France. J Invest Dermatol 2012; 132: 1998-2004.

4. Künzli K, Favre B, Chofflon M, Luca Borradori L. One gene but different proteins and diseases: the complexity of dystonin and bullous pemphigoid antigen 1. Exp Dermatol 2016; 25: 10-6.

5. Steiner-Champliaud MF, Schneider Y, Favre B, et al. BPAG1 isoform-b: complex distribution pattern in striated and heart muscle and association with plectin and alphaactinin. Exp Cell Res 2010; 316: 297-313.

6. Poliakova K, Adebola A, Leung CL, et al. BPAG1a and b associate with EB1 and EB3 and modulate vesicular transport, Golgi apparatus structure, and cell migration in C2.7 myoblasts. PloS One 2014; 9: e107535.

7. Claudepierre T, Manglapus MK, Marengi N, et al. Collagen $\mathrm{XVII}$ and BPAG1 expression in the retina: evidence for an anchoring complex in the central nervous system. J Comp Neurol 2005; 487: 190-203.

8. Teixeira VB, Cabral R, Brites MM, et al. Bullous pemphigoid and comorbidities: a case-control study in Portuguese patients. An Bras Dermatol 2014; 89: 274-9.

9. Brick KE, Weaver CH, Savica R, et al. A population-based study of the association between bullous pemphigoid and neurologic disorders. J Am Acad Dermatol 2014; 71: 1191-7.

10. Bastuji-Garin S, Joly P, Lemordant P, et al. Risk factors for bullous pemphigoid in the elderly: a prospective case-control study. J Invest Dermatol 2011; 131: 637-43.

11. Taghipour K, Chi CC, Vincent A, et al. The association of bullous pemphigoid with cerebrovascular disease and dementia: a case-control study. Arch Dermatol 2010; 146: 1251-4.

12. Hunter HJA, Hinz R, Gerhard A, et al. Brain inflammation and psoriasis: a [11C]-(R)-PK11195 positron emission tomography study. Br J Dermatol 2016; 175: 1082-4.
13. Reich K. The concept of psoriasis as a systemic inflammation: implications for disease management. J Eur Acad Dermatol Venereol 2012; 26 (Suppl. 2): 3-11.

14. Tablazon ILD, Al-Dabagh A, Davis SA, Feldman SR. Risk of cardiovascular disorders in psoriasis patients: current and future. Am J Clin Dermatol 2014; 14: 1-7.

15. Armstrong EJ, Harskamp CT, Armstrong AW. Psoriasis and major adverse cardiovascular events: a systematic review and meta-analysis of observational studies. J Am Heart Assoc 2013; 2: e000062.

16. Gisondi P, Sala F, Alessandrini F, et al. Mild cognitive impairment in patients with moderate to severe chronic plaque psoriasis. Dermatol Basel Switz 2014; 228: 78-85.

17. Dmochowski M, Neumann E, Bowszyc-Dmochowska M. A case with coexistent bullous pemphigoid and psoriasis vulgaris. Immunofluorescence and immunoblot study. Post Derm 1993; 10: 19-27.

18. Morris SD, Mallipeddi R, Oyama N, et al. Psoriasis bullosa acquisita. Clin Exp Dermatol 2002; 27: 665-9.

19. Dmochowski M, Gornowicz-Porowska J, Pietkiewicz P, Bowszyc-Dmochowska M. Are patients with anti-BP230 lgG antibodies but without anti-BP180NC16a IgG antibodies suffering from less stereotypical varieties of bullous pemphigoid? International Pre IID Satellite Symposium on Autoimmune Bullous Diseases; Lübeck, Germany, 6-7 $7^{\text {th }}$ May 2013.

20. Gornowicz-Porowska J, Pietkiewicz P, Bowszyc-Dmochowska $M$, et al. Schorzenia neurodegeneracyjne a pemfigoid pęcherzowy - retrospektywne badanie immunopatologiczne. Przegl Dermatol 2015; 102: 89-90.

21. Messingham K, Narayanan N, Aust S, et al. Collagen XVII autoantibodies are present in Parkinson's disease patients and co-localize with tyrosine hydroxylase in the substantia nigra. J Invest Dermatol 2015; 135 Suppl. 1: S14.

22. Noe MH, Messingham K, Aust S, et al. Bullous pemphigoid patients exhibit increased markers of immunoscence. 2015 Annual Meeting of the SID; Atlanta, GA, USA, 6-9 ${ }^{\text {th }}$ May 2015, Meeting Program, S1: LB738.

23. Cordel N, Chosidow O, Hellot MF, et al. French Study Group of Bullous Diseases. Neurological disorders in patients with bullous pemphigoid. Dermatol Basel Switz 2007; 215: 187-91.

24. Langan SM, Groves RW, West J. The relationship between neurological disease and bullous pemphigoid: a populationbased case-control study. J Invest Dermatol 2011; 131: 631-6.

25. Tarazona MJM, Mota ANC de M, Gripp AC, et al. Bullous pemphigoid and neurological disease: statistics from a dermatology service. An Bras Dermatol 2015; 90: 280-2.

26. Jedlickova $H$, Hlubinka M, Pavlik T, et al. Bullous pemphigoid and internal diseases - a case-control study. Eur J Dermatol 2010; 20: 96-101.

27. Sheu JJ, Wang KH, Lin HC, Huang CC. Psoriasis is associated with an increased risk of parkinsonism: a population-based 5-year follow-up study. J Am Acad Dermatol 2013; 68: 992-9.

28. Rossa G. The prevalence of Alzheimer's type dementia and vascular dementia in the district of Swiebodzin. Psychiatr Pol 1997; 31: 121-34.

29. Bonek R. Znaczenie badania autoprzeciwciał w diagnostyce autoimmunologicznych zapaleń mózgu; Jesienna Szkoła Immunologii. Euroimmun Polska Meeting; Kocierz, Targanice, Poland, $4^{\text {th }}$ September 2015.

30. Cahalan S. Brain on fire: my month of madness. Free Press, 2012.

31. Pietkiewicz P, Gornowicz-Porowska J, Bowszyc-Dmochowska M, et al. Bullous pemphigoid and neurodegenerative diseases: a study in a setting of a Central European university dermatology department. Aging Clin Exp Res 2016; 28: 659-63. 